
JERZY ZALEWSKI

\title{
Knowledge of contraceptive methods among adolescents/young adults
}

\begin{abstract}
Introduction. Family planning methods let one make an informed decision about having children at the right time. People have been trying to adopt the right family planning methods for centuries, mainly through using various contraceptives. These used to be very diverse over the time, yet the goal remained the same - preventing pregnancy.

Aim. Assessment of the factors affecting the knowledge and prevalence of contraceptive methods, as well as the factors affecting sexual behavior of adolescents/young adults.

Material and methods. 219 individuals (111 women and 108 men) between 17-21 years of age, attending secondary schools, participated in this voluntary and anonymous study. A survey questionnaire prepared by the authors was used as a study tool. Statistical analysis of the data obtained was performed using the SPSS 17.0 software.

Results. $81 \%$ of the adolescents/young adults stated that their knowledge of contraception is sufficient for them to be in charge of their own sexual lives, whilst some $9 \%$ mentioned that they lacked such knowledge, $85 \%$ of the young people studied stated that they were interested in contraception. The main sources of knowledge about contraception are the Internet (35\%) and peers (22\%). The survey showed that $59 \%$ of the individuals correctly answered the question regarding the length of a woman's menstrual cycle, $77 \%$ correctly defined the first day of the cycle, and $49 \%$ correctly determined the time of ovulation. The studied group of people had difficulties determining menstrual cycle phases; $23 \%$ considered them as fixed, 27\% - that they are variable, and 29\% gave the correct answer. The surveyed people thought that contraceptive method effectiveness (43\%), availability (33\%) and ease of administration (17\%) are most important when choosing the method. Condoms are the most common (72\%) contraceptive method among the surveyed people while hormonal contraceptives and interrupted intercourse are poorly known (12\% and $11 \%$, respectively). The respondents deemed the hormonal method (50\%), and intrauterine contraceptive methods $(21 \%)$ and condoms $(21 \%)$ the most effective modes of contraception. They thought that interrupted intercourse (53\%) and natural birth control methods (34\%) are the least effective. The age at which most of the respondents started their sexual life ranged between 16 and 18; with the age of 16 being the most common (24.2\%). Some $40 \%$ of the surveyed called their sexual intercourse occasional.

Conclusions. Adolescents consider both the Internet and their peers the primary source of information about sexuality, family planning or birth control methods. The average age of the first sexual experiences has been gradually decreasing and the condom remains to be the most common method of contraception for adolescents/young adults. The knowledge concerning family is insufficient and frequently inaccurate. Education in the fields of sexuality, contraceptive and family planning methods should be included in primary school curriculum.
\end{abstract}

Keywords: contraceptives, post middle school adolescents, start of sexual life, family.

DOI: $10.1515 /$ pjph-2015-0042

\section{INTRODUCTION}

The first research studies looking at the subjects of normal sexual development, fertility and fertilization appeared in the middle of the 19th century and they are widely popular until today, yet the first contraceptive methods appeared in prehistoric times, practically among all cultures of the world. Many of them are rooted in folk medicine while others used magic, customs or superstitions $[1,2]$. Through the recent decades, new family planning and contraception methods have been developed. In particular, the changes affected the composition of oral contraceptives, their dosage, along with the development of intrauterine or percutaneous contraceptive systems, as well as vaginal hormonal contraceptive rings. This offers the possibility of selecting the most appropriate contraceptive method from the wide array of available methods. Promoting modern, effective and safe contraception appears to be the most vital birth control method [3].

In recent years, adolescents have started to mature earlier than before, which resulted in a number of negative implications, particularly affecting their reproductive abilities 
and sexual health. Yet, the consequences may become visible after many years [4]. Both the rates of sexually transmitted diseases and the number of unplanned or undesired pregnancies in adolescents/young adults is on the rise, which calls for the need of preventing such phenomena. Thus, it is essential to offer sexual education to new generations, since this can be regarded as a preventive measure decreasing the risks related to early sexual initiation. Teaching children and the youth about sex is a part of school education, aiming at developing sexually healthy people, who accept their sex, are able to express their sexuality physically and emotionally, accept the gender roles, as well as have a sense of responsibility for their sexual conduct and the effect it may have on their partners and the rest of the society [5].

An increase in the rate of teenage pregnancies has been observed in Poland during the last 20 years [6]. This is a negative phenomenon, both because of its social and economic impact, as well as the medical implications. These teenagers achieve physical and sexual maturity at a younger age but they still do achieve neither a full emotional nor social maturity.

\section{AIM}

The aim of this study was to evaluate the adolescents/ young adults' knowledge about methods of contraception, the popularity of these, effectiveness of contraceptive methods, and check the young individuals' knowledge about family planning as well as describe sexual behavior of adolescents/young adults depending on age, gender, place of residence, type of post middle school education, family's financial status and their parents' education.

\section{MATERIAL AND METHODS}

The experimental material includes 219 surveys handed out to students during the academic year 2009/2010 at the following schools: Agnieszka Osiecka Secondary School No. 18 of Wroclaw (SS) - 77 surveys, John Paul II County School Complex located in Zmigrod (CSCZ) - 73 surveys and the Complex of Gastronomy Schools in Wroclaw (CSCW) - 69 surveys. The surveyed students fall in the 1721 year age range, with the actual numbers being as follows: 17 -year-olds -85 individuals, 18 -year-olds -60 individuals, 19-year-olds -48 individual, 20 -year-olds -25 individuals, 21 -year-old -1 individual. The participants were divided into 3 groups depending on their age: 17 -year-olds, 18 -yearolds, 19-year-olds and more, which constituted 39\%, 27\% and $34 \%$, respectively. The mean age of the respondents was 18 years and 1 month.

The survey was anonymous and voluntary. It was prepared by the authors of the study and included questions concerning the participants' age, gender, place of residence, family relationships (parents' marital status, number of siblings, having a separate room, owning a computer), parents' education level, the knowledge of female anatomy and physiology of the reproductive system, the age at which an individual partakes in sexual activities for the first time, contraceptive methods used and their effectiveness and knowledge of methods protecting against unwanted pregnancy.
The SPSS 17.0 software was used to perform a statistical analysis of the empirical data obtained. The study variables are nominal and measurements were the measurements of relationships; thus, a chi-square test was used. To measure the power of the relationship, a contingency coefficient as a symmetry measure was used. All analyses were performed on the alpha level $=0.05$. Correlations with alpha $<0.05$ were considered to be statistically significant.

\section{RESULTS}

219 students of post middle schools participated in the study, including 111 (51\%) females and 108 (49\%) males. The 17-year-olds constituted the most prevalent (39\%) age group. Regarding the place of residence, the individuals from the major city were the most prevalent (44\%), followed by those living in villages (30\%) and in small towns (26\%). In the study group, some $65 \%$ of respondents have good family situation, $20 \%$ have average and $15 \%$ have a bad family situation.

The knowledge about the female menstrual cycle is very important during the puberty period, particularly for allowing an informed use of family planning methods and contraceptives. The analysis of the study material demonstrated that providing the right answer was not easy for some of the respondents. $77 \%$ of the adolescents/young adults surveyed correctly defined the first day of the cycle as the first day of bleeding. The CSCW students (84.1\%) and the CSCZ students $(82.2 \%)$ had the least trouble determining the first day of the cycle. Women were more likely to provide correct answers $(84.7 \%)$ than men $(69.4 \%)$. Men admitted to the lack knowledge much more frequently (13.9\%) than women. $59 \%$ of the surveyed knew that a woman's menstrual cycle lasts for about one month. Small town residents' provided the majority of correct answers (77.2\%) in this case but only $29 \%$ of the participants were able to define cycle phases correctly. $47 \%$ of the surveyed group knew when ovulation occurs, and $41 \%$ knew that the ovum matures in the ovary. The period of time during which the ovum can undergo fertilization was correctly defined by $44 \%$ of the respondents, and $38 \%$ of the group surveyed stated that this period can last up to 7 days. Overall, in the case of all answers to the questions pertaining to the knowledge of the menstrual cycle, there were individuals who selected a wrong answer or gave the answer "I don't know".

Taking into account the adolescents'/young adults' knowledge of the menstrual cycle, the responses to questions concerning natural methods of birth control were mostly correct. $60 \%$ of the respondents knew what this method consists of (of body temperature monitoring and cervical mucus observation), with women providing the correct answer (76.6\%) much more frequently than men $(43.5 \%)$. On the other hand, men stated much more frequently their lack of knowledge both in reference to the nature of the natural birth control methods and regarding the possibility of distinguishing phases of the menstrual cycle based on body temperature monitoring. The $\mathrm{CSCW}$ students most frequently (60.9\%) stated that body temperature monitoring allows to distinguish fertile and non-fertile phases of the menstrual cycle, while the CSCZ students most frequently admitted their lack of knowledge. 
Most respondents (91\%) knew that a woman may conceive during her first sexual intercourse, while $9 \%$ believed that the first sexual intercourse cannot lead to conception.

It was the question regarding the location of the uterus (namely "Where is the uterus") that elicited the most surprising answers. Only $87 \%$ of the respondents provided a correct answer: $7 \%$ believed that it is near the belly button, $1 \%$ - that it is above the belly button, and 5\% did not know the answer at all. Incorrect answers were most frequently provided by males and SS students.

Some $54 \%$ of the study participants had already had sexual relations, with the mean age of the first sexual intercourse being 16 years and 4 months. Some $3.2 \%$ of the surveyed stated that their first sexual intercourse was at the age of 14 , and most frequently the first sexual intercourse occurred at the age between 16 and 18 years.

Some $46 \%$ of the surveyed individuals said that the beginning of their sexual life was at the same time as that of most of their peers and $40 \%$ of admitted to engaging in occasional sexual activity, regardless of their age, gender or school type. Women (57.7\%), 17-year-olds (62.4\%) and the SS (61\%) and CSCZ students (47.9\%) more frequently stated that they had not had a sexual intercourse yet. There was no question regarding whether the partner was steady or occasional, but one might assume that occasional sexual encounters occurred with a person whom the respondent had met by chance. The subjects who had engaged in sexual activities at an earlier age were at a higher risk of contracting sexually transmitted diseases because of the possibility that multiple partners had been involved. It needs emphasizing that adolescents/young adults most frequently have their first sexual intercourse between 16 and 18 years of age, when they are post primary school students. Thus, it is extremely important to provide them with reliable and accurate information about sexuality. Classes on that should be included in the primary school curriculum, so as to educate them before their very first sexual encounter.

Some $42 \%$ of the sexually active adolescents/youth admit to use contraceptives all the time. There are also few individuals using these occasionally $(9 \%)$ or not using them at all (3\%). Most respondents (72\%), regardless of the type of school they attended, pointed to the condom as their favorite contraception method. There were also hormonal contraceptive methods $(12 \%)$ and interrupted intercourse $(11 \%)$, which were most frequently mentioned by CSCW students. Women $(66.7 \%)$ and men $(77.8 \%)$ most frequently stated that they regard the condom as the preferred contraceptive method.

Adolescents/young adults tend to believe that hormonal contraceptive methods are the most effective (50\%). Others pointed to condoms $(21 \%)$ and intrauterine contraceptive devices $(21 \%)$ were deemed less effective by both women $(59.5 \%)$ and men $(39.8 \%)$. These findings imply that the adolescents/young adults regarded hormonal methods as the most effective. Yet, only some $49 \%$ of them understood the way contraceptive pills work, with $22 \%$ convinced that they are spermicidal, and 19\% believing that they induce an early abortion.

The least effective contraceptive methods, according to the adolescents/young adults surveyed, include interrupted intercourse (53\%) and natural birth control methods (34\%), regardless of the age, place of residence or the school type.

The majority of the respondents (48\%) are aware that the condom may not prevent pregnancy and sexually transmitted diseases. Small town residents were among those who gave the correct answers most frequently (77.2\%) while those who live in a major city $(34.4 \%)$ or in villages $(42.4 \%)$ provided more varied answers which may reflect their lack of knowledge.

Adolescents/young adults believe that choosing a birth control method should depend on its effectiveness (43\%), availability (33\%) and ease of use (17\%).

They regarded their knowledge as very extensive but the findings show that it is, unfortunately, very superficial. $81 \%$ of the individuals surveyed stated that their knowledge of contraceptive methods is sufficiently extensive to control their sexual life, and $10 \%$ thought that their knowledge is broad and covers all contraceptive methods and means. The remaining individuals lacked such knowledge. The broad knowledge declared by the participants is usually not confirmed by detailed questions. $41 \%$ believed that the pre-ejaculatory fluid does not contain sperm, and 5\% did not know the answer to this question. The response "no" was the most frequent among the CSCZ students (47.9\%) and among the 18-year-olds (55\%).

The respondents pointed to the Internet $(35 \%)$ and their peers $(22 \%)$ as the primary sources of information about contraception. Books and flyers $(9 \%)$, as well as the press or television were less common $(8 \%)$. Surprisingly, parents $(8 \%)$, school $(6 \%)$ and physicians $(4 \%)$ were cited by a relatively low percentage of respondents (the number of responses was not equal to the number of the individuals surveyed since the participants could have chosen more than one answer). Family should be the first reliable source of information about all the matters related to sex. However, some $64 \%$ of the adolescents/young adults participating in the study stated that they had never had an open talk with their parents about anything related to sexual development. Neither women (55\%) nor men (72.2\%) discussed any sexrelated subjects they were interested in. Only $16.4 \%$ of the surveyed individuals decided to talk to parents about it. Usually, students of all schools said that they did not talk to their parents $(63.5 \%)$, and $28.6 \%$ of the SS students could talk openly with their parents. An analysis of the material looking at the relationship between one's situation at home (or a complete lack of family,) and the types of responses given, was conducted. It was done taking into account the family's financial situation and parents' education. The obtained data indicates that adolescents/young adults' knowledge does not depend on any of these factors. Some $85 \%$ of the group members were interested in subjects associated with contraception, yet they chose not to discuss any subjects related to sex, puberty and contraceptives with their parents. At the same time, parents were unwilling to accept the fact that their child is sexually active. Postponing the discussion or even avoiding it totally will not eradicate the problem, since it is better to start to be sexually active having an appropriate knowledge. Discussing contraceptives is impossible without talking about sex. These subjects are inseparable and the subject of sexuality should not be avoided at home. Only having a broad 
and reliable knowledge about contraceptives allows would young people to make an informed and correct choice.

Only $51 \%$ of the surveyed women see a gynecologist regularly, and $25 \%$ does not see a gynecologist on a regular basis. Women from the major city (38.6\%), female CSCW students of Wroclaw $(38.6 \%)$ and women in poor financial circumstances $(55 \%)$ were more likely to frequent gynecologists regularly, while women who live in a small town or village, individuals with good or average financial status, as well as students of other schools mentioned in the study, state that they have yet to see a gynecologist. This implies that women in a large city see gynecologist more often than those in smaller agglomerations. This may be caused by higher awareness and sense of responsibility for one's own health, better and more anonymous access to health care facilities.

\section{DISCUSSION}

The findings of the study show that most participants lack knowledge about the female menstrual cycle and only a few of them are able to admit it. Most believe that they are competent in this area. Both men and women, regardless of their place of residence, age, financial conditions or parents' education, have difficulties describing the phases of the menstrual cycle, defining the first day of the cycle and sometimes ovulation. The analysis of the responses to the questions concerning the female sexual cycle demonstrates that at this stage and with that kind of knowledge, a significant portion of adolescents/young adults cannot afford to think that their information is sufficient to control their sexual life in an informed way. H. Huras et al. also showed that adolescents/young adults are not knowledgeable in the area of the menstrual cycle physiology, and are not able to define the first day of the cycle or specify the day of ovulation" [7].

Many adolescents/young adults who start their sexual life as teenagers are not aware of negative consequences which may be associated with irresponsible sexual conduct. To a large extent, this results from the lack of comprehensive knowledge about sexuality in general. Proper sex education should ensure solid knowledge of fertility mechanisms and their regulation and should be supported by parental sex education. The study demonstrated that the Internet and peers had the greatest impact on the knowledge about sex, while school and family contributed little. This is not consistent with the study conducted by Filipp et al.; its goal was to evaluate family planning methods among teenagers in Warsaw in which it was shown that school was the primary source of knowledge among younger teenagers (15-18 years old) and a gynecologist was cited most frequently by older (18-19 years old) respondents [8]. The present study confirmed the findings of Nowosielski et al. looking at the patterns of sexuality among young adults living in Silesian Province. In that case, it was the media that served as the primary source of information while physicians and parents were less common cited as sources of knowledge [4]. The adolescent/young adult participants in the survey conducted by Huras H., pointed to the school as the primary source of information, followed by the media and their peers.
Literature and health care system were cited by a small percentage of the surveyed [7]. This might depend on the place of residence, on parents' education and knowledge, and, most of all, on the teaching standards provided by educators conducting classes dedicated to sexuality education, in Poland known as "preparation for family life." Thus, it is essential to introduce sex education adhering to the highest standards at school. In addition, parents should be able to become acquainted with the subjects taught and statistics on sexual behavior of adolescents/young adults. The increase in parental knowledge would spur more parents to allow their children to participate in such classes. On the other hand, the higher the level of such classes, the more eager the adolescents/young adults would be to participate in these.

Currently, the average age of sexual initiation is continually lowering. On average, it has started decreasing by 2-3 years since 1960 in the entire Western Europe and slightly later in Eastern Europe. Currently, an average age of first sexual intercourse in Western and Central Europe is 17-18 years of age, while in Eastern Europe it is approximately 20 years. The studies performed among the 19-year-olds showed that $52.5 \%$ of girls and $61.6 \%$ of boys had already started their sexual life [8]. In this study, $54 \%$ of the participants are reported to have already have a sexual intercourse, with the mean age of the first sexual intercourse 16 years and 4 months. The age range of the first sexual experiences was most frequently between 16 and 18 years of age. This confirms the findings of Sajdak S. et al. in their study looking at sexual initiation [9]. Filipp E. et al. determined that among women 18-19 years of age, the mean age of the first intercourse was 16.76 [8] and in the study of the 18-year-olds by Woynarowska B. et al. the mean age in which sexual life was started was 16.8 for girls and 16.5 for boys [10].

Among the adolescents/young adults who are sexually active, only $42 \%$ maintain that they use contraceptives, while a small part of the surveyed uses them only occasionally $(9 \%)$ or does not use them at all (3\%). Filippe E. et al. in his studies demonstrated that $20 \%$ of young women did not use any birth control method and in the studies of Woynarowska B. et al.11.1\% of the respondents did not use any contraceptive method during their last sexual intercourse, while 5.2\% were unsure about it $[8,10]$.

Although numerous contraceptives were available, no single study participant decided to check more than one. According to the studies of Woynarowska B. et al. on sexual behaviour of teenagers 16 and 18 years old in Poland in 2005 [10] and the studies of Plocka-Molinska M. concerning contraceptive knowledge and use among secondary school students [11], the condom was the most frequently used contraceptive, followed by contraceptive pills. Nowosielski also showed that the condom was the most common contraceptive method [4]. The studies of Jakimiuk J. on the use of contraceptives by adolescents [6] and of Filipp E. et al. on the birth control methods among teenage females [8] confirmed that the condom is a common contraceptive method in adolescents/young adults between 16-20 years of age. The studies looking at American teenagers between 15 and 19 years of age do not confirm these findings, since they showed that $30 \%$ of teenagers use various contraceptive methods and measures, including, in the descending order 
of frequency: oral hormonal contraceptives, the condom, hormonal intramuscular injections, and, less often, interrupted intercourse, implants and cervical caps [7].

The findings mentioned above are based on the statements elicited from the adolescents/young adults and they might not be accurate enough. Also, this study included only a small number of participants which means that these findings cannot be used for the entire Polish adolescent/young adult population. To get a bigger picture, there is a need for a study looking at adolescents/young adults from other regions of Poland. This study will be continued.

The results of this study lead to the conclusion that the knowledge about methods of contraception, how to use them, their effectiveness or sexual behavior patterns are not dependent on the age, gender, place of residence, type of post junior high school, family financial circumstances and parents' education. The primary sources of knowledge about sex life and family planning or contraceptive methods are the Internet and peers. Family, school or sex health specialists are less common sources of knowledge. A mean age of the start of sexual life has been gradually decreasing. Family planning and contraception knowledge of adolescents/young adults is insufficient, and frequently inaccurate. The education of sex, contraception and family planning method knowledge should start in primary school.

\section{REFERENCES}

1. Jarząbek G, Pawelczyk M, Friebe Z. History of family planning and contraceptive methods. Ginekol Prakt. 2006;3:30-3.

2. Kiełbasińska J, Dmoch-Gejzerska E. History of conception regulation. Poł Nauk Prakt. 2008;1:50-2.

3. Złotowicz-Grochowska R. Contemporary hormonal contraceptives. Farmakol Farmakoter. 2007;8:33-7.

4. Nowosielski K, Drosdzol A, Skrzypulec V, et al. Sex in young adults of Silesian Province. Ginekol Parakt. 2005;2:32-39.

5. Woynarowska B. Health Education. Warszawa: Wydawnictwo Naukowe PWN; 2008.

6. Jakimiuk A, Nowicka M. Contraceptives in adolescents. Ginekol po Dyplomie. 2007;4:28-41.

7. Huras H, Prażmowska B, Klimek M. Middle school student knowledge level of family planning methods. Poł Nauk Prakt. 2009;4:8-11.

8. Filipp E, Pawłowska A, Wilczyńska A, et al. Family planning methods among female teenagers. Gin Prakt. 2005;4:46-52.

9. Sajdak S, Łukaszczyk I, Ziętkowski W. Evaluation of the start of adolescence sexual presently. Med Wieku Rozw. 2000;2:177-83.

10. Woynowska B, Małakowska A, Tabak I. Sexual behaviour among 16and 18-year-old adolescents in Poland in 2005. Ginekol Pol.2006;9:66777.

11. Płocka-Molińska M, Moś K. Knowledge and use of contraceptive methods among secondary school students. Pol Med Rodz. 2004;1:465-7.

\section{Corresponding author}

Justyna Surdyka

Faculty of Health Science, Wroclaw Medical University, Poland

5 K. Bartla Str., 51-618 Wrocław, Poland

tel.: +48 518-374-616

E-mail: justyna.surdyka@o2.pl 